\title{
Patient Awareness of Head and Neck Cancer Risk Factors: Assessment of the General Otolaryngology Population
}

\author{
Carolyn DeBiase ${ }^{1}$, Garrett $\mathrm{Ni}^{2}$, Neil Gildener-Leapman ${ }^{3}$ and Lisa Galati ${ }^{4}$ \\ ${ }^{1}$ Department of Otolaryngology - Head \& Neck Surgery, Mayo Clinic Arizona, Phoenix AZ. \\ ${ }^{2}$ Department of Otolaryngology - Head \& Neck Surgery, Lewis Katz School of Medicine at Temple University, Philadelphia PA. \\ ${ }^{3}$ Department of Otolaryngology, Albany Medical Center, Albany NY. \\ ${ }^{4}$ Associate Professor, Department of Otolaryngology - Head \& Neck Surgery, Albany Medical Center, Albany NY.
}

Corresponding Author: Garrett Ni, MD, Department of Otolaryngology - Head \& Neck Surgery, Lewis Katz, School of Medicine at Temple University, Philadelphia PA.

Received date: January 27, 2021: Accepted date: February 25, 2021: Published date: July 03,2021

Citation: DeBiase C, Garrett Ni, Neil G Leapman and Galati L. (2021) Patient Awareness of Head and Neck Cancer Risk Factors: Assessment of the General Otolaryngology Population. J. of Clinical Otorhi 3(3); DOI: 10.31579/2692-9562/021

Copyright: (C) 2021, Garrett Ni. This is an open access article distributed under the Creative Commons Attribution License, which permits unrestricted use, distribution, and reproduction in any medium, provided the original work is properly cited.

\begin{abstract}
Objective: The general public's knowledge of the risk factors for head and neck cancer is insufficient. The level of awareness of such risk factors amongst the otolaryngology clinic patient population has not yet been elucidated in the literature.

Method: This individual cohort study took place at a tertiary academic center. A survey was designed and administered to all patients who presented to otolaryngology clinic from 2017 to 2018 to assess knowledge of head and neck cancer risk factors. The main outcome measures were percentage of correct responses for each cancer risk factor and comparison of percent correct between cancer and non-cancer patients.

Results: A total of 510 patients were enrolled in the study including 69 patients $(13.5 \%)$ with a history of head and neck cancer. The most well recognized risk factors by all patients were cigarettes $(83.7 \%)$ and chewing tobacco $(77.5 \%)$. Twenty-nine percent of patients correctly identified alcohol as a risk factor. Additional risk factors were poorly recognized. Cancer patients had a similar or better correct response rate than non-cancer patients except for chewing tobacco ( $68.1 \%$ vs $78.9 \%$ respectively).

Conclusion: The general otolaryngology clinic population, especially patients with a history of head and neck cancer, demonstrated improved knowledge of some risk factors for head and neck cancer, but insufficient awareness of alcohol and HPV transmissible behaviors.
\end{abstract}

Key Words: head and neck, health policy, larynx, oral cavity, oropharynx

\section{Introduction}

Head and neck cancer is a significant cause of morbidity and mortality in the United States, with an estimated 63,030 new cases and 13,360 deaths of oral cavity, pharynx, and larynx cancer in 2017 [1]. The general population knowledge of head and neck cancer and its risk factors is poor [2]. The general population is most familiar with smoking as a risk factor for head and neck cancer (HNC), with $54-65 \%$ correctly identifying smoking as a risk factor [2-4]. With the rise in Human Papilloma Virus (HPV) related cancers of the head and neck, public awareness of this viral risk factor has increased but is still only at $12-28 \%$ [2-4]. One of our main concerns and the motivation for pursuing this study is the lack of knowledge of alcohol as a risk factor for HNC that we have seen among our own patients. Previous surveys of the adult population show a wide range of awareness, with $4.8-40 \%$ of surveyed adults aware that alcohol is a risk factor for HNC [2-4]. The knowledge of the general otolaryngology patient population regarding head and neck cancer risk factors is currently unknown. Previous United States studies have focused on college students, patients presenting to free cancer screening programs,
American Indians, rural populations, predominantly black populations, and the general public [2-7].

The purpose of this study was to assess the baseline awareness of the risk factors for head and neck cancer among the general otolaryngology clinic population in an academic setting in the greater Albany, NY area. We hypothesized that the otolaryngology patient population will have better knowledge of head and neck cancer risk factors, compared to the general population as surveyed by Luryi at al. In addition, patients already diagnosed with head and neck cancer at time of survey are expected to have better awareness of HNC risk factors compared to the general otolaryngology patient population without head and neck cancer.

\section{Methods}

This study was exempt by the Albany Medical College Institutional Review Board as an anonymous survey with no patient identifiers collected. All patients presented to their outpatient otolaryngology clinic appointment between 2017 and 2018 were given a survey with attached informational sheet describing the study. Clinic staff were instructed to 
advise patients that we were conducting a survey to assess head and neck cancer awareness and that patient participation was voluntary. Completed surveys were collected by clinic staff prior to check-out. Survey answers and educational information were displayed at the check-out desk to prevent them being seen prior to completing the survey. Surveys were later numbered for record keeping in a randomized blocking schedule.

Inclusion criteria of our study were the ability to read and write in the English language and willingness to participate in the survey. Albany County is predominantly English-speaking (86\%) and this is reflected in our clinic population. ${ }^{8}$ Patients were excluded if they did not answer the personal history of head and neck cancer question or if they made annotations on the survey indicating that they did not understand the survey. The goal of patient accrual was 500 study participants with at least 50 participant with a positive history of head and neck cancer to reach statistical power. Our survey consists of 19 factors including microwaved plastic, not-brushing teeth, smoking marijuana, artificial sweeteners, chewing tobacco, kissing, cocaine, oral sex, smoking cigarettes, heroin, methamphetamines, alcoholic drinks, eating spicy food, acid reflux, salty foods, chewing betel quid, mouth wash, electronic cigarettes, and eating marijuana (Figure 1). The 19 factors are partially based off of prior survey studies while others are frequently asked by patients regarding their carcinogenic potential in our otolaryngology clinics. Our survey asked patients to identify which of 19 factors had been scientifically proven to contribute to head and neck cancer. Patients were instructed prior to starting the survey that not all factors listed were contributors to head and neck cancer. Patients were additionally asked if this was their first time completing the survey, if they had been previously diagnosed with head and neck cancer, and their reason for otolaryngology appointment.

\section{Patient Survey on Risk Factors for Head and Neck (Mouth/Throat/Voice box) Cancer}

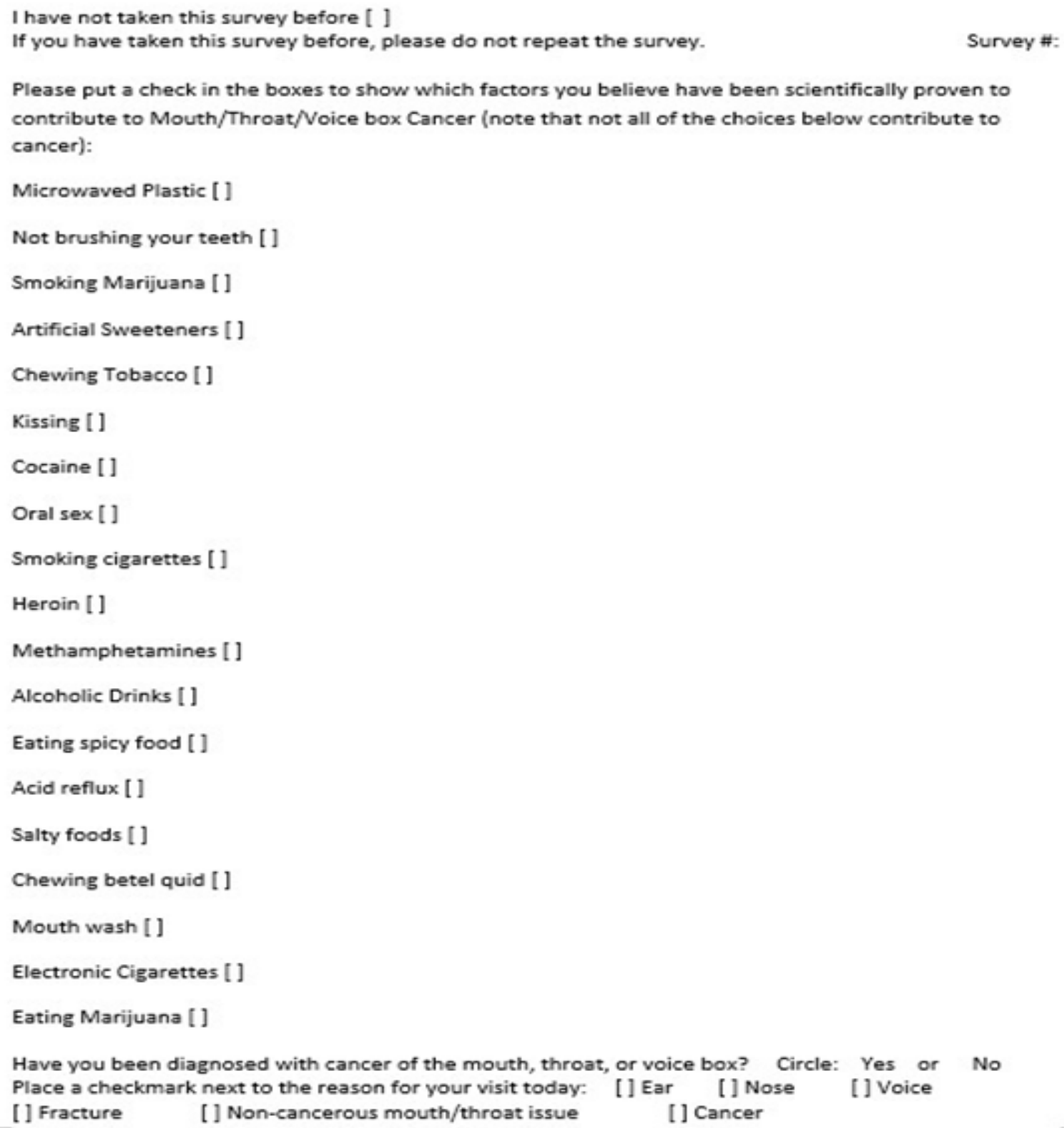


All surveys that met inclusion criteria were entered into Microsoft Excel for data analysis. Demographic data including prior diagnosis of head and neck cancer and reason for visit (primary complaint) were tabulated. Percentage of patients correctly identifying each item as a contributor or non-contributor to head and neck cancer was calculated and compared between the cancer patients and non-cancer patients using chi-square analysis. A standard $\mathrm{p}$ value of less than 0.05 was used to determine significance. The data analysis that support the findings of this study are available from the corresponding author upon reasonable request. Acid reflux was omitted from analysis due to inconsistent data in the current literature on its causality to head and neck cancer.

\section{Results}

Over a 14-month period, 510 patients completed the survey and fulfilled inclusion criteria. The majority of patients $(86.5 \%)$ had no history of head and neck cancer (Table 1). The most common reason for clinic visit was an ear primary complaint ( $\mathrm{N}=292,57.43 \%$ of total patients) (Table 2). This was followed by voice issue $(\mathrm{N}=176,34.5 \%)$ and non-cancerous mouth/throat issue $(\mathrm{N}=139,27.3 \%)$. Patients responded with multiple primary complaints and as such the total $\mathrm{N}$ in Table 2 is 830 , however, percentages were calculated based on total number of patients $(510)$.

\begin{tabular}{|l|l|l|}
\hline & N & \% of total patients \\
\hline Positive & 69 & $13.5 \%$ \\
\hline Negative & 441 & $86.5 \%$ \\
\hline Total & 510 & \\
\hline
\end{tabular}

Table 1. Prior history of head and neck cancer

\begin{tabular}{|l|l|l|}
\hline & $\mathbf{N}$ & \% of total patients \\
\hline Ear & 292 & $57.3 \%$ \\
\hline Voice & 176 & $34.5 \%$ \\
\hline Non-cancerous mouth/throat & 139 & $27.3 \%$ \\
\hline Nose & 99 & $19.4 \%$ \\
\hline Cancer & 67 & $13.1 \%$ \\
\hline No answer & 48 & $9.4 \%$ \\
\hline Fracture & 9 & $1.8 \%$ \\
\hline TOTAL & 830 & \\
\hline
\end{tabular}

Table 2. Reason for clinic visit

\begin{tabular}{|c|c|c|c|c|}
\hline FACTOR & $\begin{array}{c}\text { TOTAL PATIENTS } \\
\text { CORRECT (\%) }\end{array}$ & $\begin{array}{c}\text { CANCER PTS } \\
\text { CORRECT (\%) }\end{array}$ & $\begin{array}{c}\text { NON-CA PTS } \\
\text { CORRECT (\%) }\end{array}$ & P VALUE \\
\hline Microwaved plastic & $354(69.4 \%)$ & $47(68.1 \%)$ & $307(69.6 \%)$ & 0.802 \\
\hline Not brushing teeth & $134(26.3 \%)$ & $17(24.6 \%)$ & $117(26.5 \%)$ & 0.740 \\
\hline Smoking marijuana & $335(65.7 \%)$ & $44(63.8 \%)$ & $291(66.0 \%)$ & 0.718 \\
\hline Artificial sweeteners & $378(74.1 \%)$ & $51(73.9 \%)$ & $327(74.1 \%)$ & 0.967 \\
\hline Chewing tobacco & $395(77.5 \%)$ & $47(68.1 \%)$ & $348(78.9 \%)$ & $\mathbf{0 . 0 4 6} *$ \\
\hline Kissing & $481(94.3 \%)$ & $62(89.9 \%)$ & $419(95.0 \%)$ & 0.085 \\
\hline Cocaine & $368(72.2 \%)$ & $57(82.6 \%)$ & $311(70.5 \%)$ & $\mathbf{0 . 0 3 7} *$ \\
\hline Oral sex & $111(21.8 \%)$ & $23(33.3 \%)$ & $88(20.0 \%)$ & $\mathbf{0 . 0 1 2} *$ \\
\hline Smoking cigarettes & $427(83.7 \%)$ & $59(85.5 \%)$ & $368(83.4 \%)$ & 0.666 \\
\hline Heroin & $396(77.7 \%)$ & $60(87.0 \%)$ & $336(76.2 \%)$ & $\mathbf{0 . 0 4 6} *$ \\
\hline Methamphetamines & $379(74.3 \%)$ & $61(88.4 \%)$ & $318(72.1 \%)$ & $\mathbf{0 . 0 0 4} *$ \\
\hline Alcohol & $148(29.0 \%)$ & $28(40.6 \%)$ & $120(27.2 \%)$ & $\mathbf{0 . 0 2 3} *$ \\
\hline Spicy food & $469(90.2 \%)$ & $62(89.9 \%)$ & $398(90.2 \%)$ & 0.918 \\
\hline Salty foods & $473(92.7 \%)$ & $63(91.3 \%)$ & $410(93.0 \%)$ & 0.620 \\
\hline Betel quid & $74(14.5 \%)$ & $9(13.0 \%)$ & $65(14.7 \%)$ & 0.710 \\
\hline Mouthwash & $31(6.1 \%)$ & $5(7.2 \%)$ & $26(5.9 \%)$ & 0.662 \\
\hline E-cigarettes & $293(57.5 \%)$ & $44(63.8 \%)$ & $249(56.5 \%)$ & 0.254 \\
\hline Eating marijuana & $432(84.7 \%)$ & $60(87.0 \%)$ & $372(84.4 \%)$ & 0.576 \\
\hline
\end{tabular}

Table 3. Survey answers risk factors for head and neck cancer

Our primary outcome was to assess the baseline awareness of head and neck cancer (HNC) risk factors among the general otolaryngology patient population. The most well recognized risk factors were smoking cigarettes and chewing tobacco with $83.7 \%$ and $77.5 \%$ of patients with correct survey answers, respectively (Table 3 ). Only $29.0 \%$ of patients identified alcohol as a risk factor for HNC. Patients had little knowledge of the other positive risk factors: not brushing teeth $(26.3 \%)$, oral sex $(21.8 \%)$, betel quid $(14.5 \%)$, and mouthwash $(6.1 \%)$. The survey contained additional factors that have not been proven to be a positive risk factor for head and neck cancer in the most recent literature. These factors, 
i.e. non-risk factors, included microwaved plastic, smoking marijuana, artificial sweeteners, kissing, cocaine, heroin, methamphetamines, spicy foods, salty foods, e-cigarettes, and eating marijuana. The majority of patients correctly identified these non-risk factors as not contributing to HNC. However, $42.5 \%$ of patients believed e-cigarettes have a carcinogenic effect in HNC.

Our secondary goal in this study was to assess the difference in risk factor knowledge between patients with a history of HNC and those without. There were 69 patients with a history of HNC that completed the survey. Of HNC patients, $40.6 \%$ of patients identified alcohol as a risk factor compared to $27.2 \%$ of non-HNC patients (Table 3 ). HNC patients more frequently identified oral sex as a risk factor for head and neck cancer (33.3\% vs $20.0 \%$ ). Drugs with no causative link to HNC including heroin, methamphetamines, and cocaine were more often correctly identified as non-carcinogenic factors by cancer patients (Table 3). However, noncancer patients more often correctly reported chewing tobacco as a risk factor $(78.9 \%$ ) compared to $68.1 \%$ of $\mathrm{HNC}$ patients. The remainder of the risk factors did not show a statistically significant difference in answers between cancer and non-cancer patients.

\section{Discussion}

There have been increasing efforts in recent decades to improve health literacy among various patient populations. Previous studies have shown that the American public is most aware of tobacco as a risk factor for head and neck cancer and marginally aware of other risk factors [2]. We aimed to identify the level of knowledge of the general otolaryngology clinic population at a tertiary academic medical center. When comparing between the cancer and the non-cancer patients' awareness of cancer risk factors, we found that they were similar in awareness across most risk factors with the exception of chewing tobacco, cocaine use, heroin use, methamphetamine use, alcohol consumption, and oral sex. Our cancer patients have better awareness of these risk factors except for chewing tobacco which the non-cancer patient population had higher level of awareness. Our patients had a moderately high awareness of cigarettes $(83.7 \%)$ and chewing tobacco $(77.5 \%)$ as risk factors for HNC. In an online survey of the adult American public by Luryi et al, only $54.5 \%$ and $32.7 \%$ of adults correctly identified smoking cigarettes and chewing tobacco as risk factors for $\mathrm{HNC}$, respectively [2]. The increased awareness of the carcinogenic nature of tobacco observed in our population could be attributed to a general increased health literacy in patients presenting to a specialty clinic or to increased media representation of the harmful effects of tobacco in recent years. Our noncancer patient population more often correctly identified chewing tobacco as a risk factor for $\mathrm{HNC}$ compared to our cancer patients $(78.9 \%$ vs $68.1 \%$ ). Chewing tobacco is not as common in the northeast as compared to the Midwest United States [9]. Our patients were decently informed of the carcinogenic effects of chewing tobacco despite the low prevalence of this practice in our geographic location.

Drinking three or more alcoholic beverages a day increases a person's risk of developing head and neck cancer [10]. This risk is further intensified in patients who smoke tobacco and drink alcohol due to alcohol's potential solvent properties [10]. We have observed a lack of awareness especially among newly diagnosed head and neck cancer patients of the carcinogenic effects of alcohol. This was our driving force to pursue this study and bring attention to this knowledge gap. Our patients had mildly increased knowledge of alcohol as a risk factor for HNC (29.0\%) compared to the general population as surveyed by Luryi et al (4.8\%) [2]. Previous studies at cancer screening events have shown similar results to ours, with $39.5 \%$ of patients identifying alcohol as a risk factor [4]. Our cancer patients were more knowledgeable of alcohol as a risk factor $(40.6 \%)$ compared to our non-cancer patients $(27.2 \%)$. However, the awareness level is too low to consider our cancer cohort or general otolaryngology population well informed. Our faculty and house staff have made efforts to educate newly diagnosed cancer patients of the carcinogenic effects of alcohol. As evidenced from this study, more work is needed in addition to expanding our education efforts to the remainder of our clinic population. Overall, the American public and otolaryngology patient population knowledge of alcohol as a risk factor for $\mathrm{HNC}$ is poor.

With the rise in Human Papilloma Virus (HPV) related cancers of the head and neck, public awareness of this viral risk factor has increased but is still only at $12-28 \%$ [2-4]. Oral sex, increased number of sexual partners, and early age at first sexual encounter are associated with an increased risk of HNC [11]. Similar to previous studies, only $21.8 \%$ of our patients identified oral sex as a risk factor for HNC. Public awareness of HPV is increasing, but it's relation to head and neck cancer is still largely unknown by the general ENT population. Our cancer population was slightly more informed than the general ENT population (33.3\% vs $20 \%$ ). Sexual behaviors and their risk of HPV transmission is always a topic of education for our newly diagnosed HPV positive cancers patients.

It is not unexpected that the remainder of the risk factors for HNC were not commonly known by our patients. Increased risk of HNC with long term frequent use of mouthwash is thought to be related to the alcohol content [12]. Poor oral hygiene and infrequent teeth brushing has repeatedly been shown to elevate the risk of HNC [13]. Betel quid has been associated with HNC regardless of whether tobacco is added to the mixture [14]. Knowledge of these risk factors and their relation to head and neck cancer has not been well studied in the American public.

This study is the first to explore the general otolaryngology clinic patient population knowledge of head and neck cancer. We hypothesized that general otolaryngology patients would have improved knowledge compared to the general public. Our patients did show increased knowledge of the carcinogenic risk of smoking cigarettes and chewing tobacco compared to the general population as surveyed by Luryi. Our patients had improved, but still unsatisfactory, knowledge of alcohol as a risk factor for HNC. Our study brings attention to the need for patient education of the risk factors for head and neck cancer especially alcohol - a modifiable risk factor. Patients with a history of head and neck cancer are mildly more informed of the risk factors for HNC likely due to our physician to patient educational efforts, but there is still much needed improvement in this population as well.

Limitations of this study include single academic institution and a skewed demographic that does not represent the demographic of the United States. Albany county has a small Hispanic population (6\%) compared to the United States as a whole $(18 \%) .{ }^{8}$ However, as a tertiary referral center, we have a wide service area including 25 counties in New York, Vermont, and Massachusetts. This wide catch area allows a more diverse patient population to be recruited for our study. Collecting sociodemographic information would have added to our study's generalizability. Our future efforts will be aimed at improving knowledge of all otolaryngology patients through increased physician to patient efforts and educational campaigns.

\section{Conclusion}

The general otolaryngology clinic population, especially patients with a history of head and neck cancer, demonstrated improved knowledge of some risk factors for head and neck cancer, but insufficient awareness of alcohol and HPV transmissible behaviors.

\section{Acknowledgement:}

There was no grant or other support for this manuscript.

\section{Conflict of Interest:}

The authors have no disclosures. 


\section{Prior Meeting information:}

None

\section{Funding and Conflict of Interest:}

None

\section{Reference}

1. Siegel RL, Miller KD, Jemal A. (2017) Cancer Statistics. CA Cancer J Clin. 67(1):7-30.

2. Luryi AL, Yarbrough WG, Niccolai LM, et al. (2014) Public awareness of head and neck cancers: a cross-sectional survey. JAMA Otolaryngol Head Neck Surg. 140(7):639-646.

3. Merten JW, Parker A, Williams A, et al. (2016) Cancer Risk Factor Knowledge among Young Adults. J Cancer Educ.

4. Berger MH, Cohen ER, Shamrock AG, et al. (2017) Assessment of human papillomavirus awareness in association with head and neck cancer at a screening event. The Laryngoscope.

5. Dwojak S, Deschler D, Sargent M, et al. (2015) Knowledge and screening of head and neck cancer among American Indians in South Dakota. Am J Public Health. 105(6):1155-1160.

6. Riley JL, Pomery EA, Dodd VJ, et al. (2013) Disparities in knowledge of mouth or throat cancer among rural Floridians. $J$ Rural Health. 29(3):294-303.

7. Osazuwa-Peters N, Adjei Boakye E, Chen BY, et al. (2017) Sociodemographic Factors Associated With Knowledge and Risk Perception of Human Papillomavirus and Human Papillomavirus-
Associated Oropharyngeal Squamous Cell Carcinoma Among a Predominantly Black Population. JAMA Otolaryngol Head Neck Surg. 143(2):117-124

8. American Community Survey 1-year estimates. U.S. Census Bureau (2017).

9. Wang TW, Asman K, Gentzke AS, et al. (2018) Tobacco Product Use Among Adults - United States, 2017. MMWR Morb Mortal Wkly Rep. 67(44):1225-1232.

10. Kawakita D, Matsuo K. (2017) Alcohol and head and neck cancer. Cancer Metastasis Rev.

11. Shah A, Malik A, Garg A, et al. (2017) Oral sex and human papilloma virus-related head and neck squamous cell cancer: a review of the literature. Postgrad Med J.

12. Boffetta P, Hayes RB, Sartori S, et al. (2016) Mouthwash use and cancer of the head and neck: a pooled analysis from the International Head and Neck Cancer Epidemiology Consortium. Eur J Cancer Prev. 25(4):344-348.

13. Ahrens W, Pohlabeln H, Foraita R, et al. (2014) Oral health, dental care and mouthwash associated with upper aerodigestive tract cancer risk in Europe: the ARCAGE study. Oral Oncol. 50(6):616-625.

14. Gupta B, Johnson NW. (2014) Systematic review and metaanalysis of association of smokeless tobacco and of betel quid without tobacco with incidence of oral cancer in South Asia and the Pacific. PLoS One. 9(11):e113385.
This work is licensed under Creative Commons Attribution 4.0 License

To Submit Your Article Click Here: Submit Manuscript

DOI: $10.31579 / 2692-9562 / 021$
Ready to submit your research? Choose Auctores and benefit from:

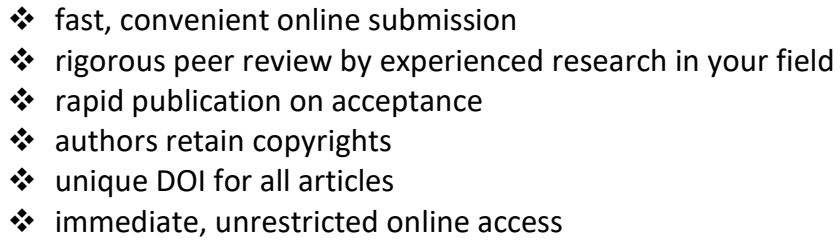

At Auctores, research is always in progress.

Learn more www.auctoresonline.org/journals/journal-of-clinicalotorhinolaryngology 